\title{
RIESZ MATRICES THAT ARE ALSO HAUSDORFF MATRICES
}

\author{
C. E. HARRELL
}

If $A=\left(a_{0}, a_{1}, a_{2}, \cdots\right)$ is an infinite sequence, $a_{0}>0, a_{p} \geqq 0$ for $p \geqq 1$ and $S$ is the sequence of partial sums of $A$ then by the Riesz matrix for $A$ we shall mean the infinite triangular matrix [A] such that $[A]_{n p}=a_{p} / S_{n}, 0 \leqq p \leqq n$ and $[A]_{n p}=0$ otherwise.

If $D=\left(d_{0}, d_{1}, d_{2}, \cdots\right)$ is an infinite number sequence, $\Delta^{0} d_{p}=d_{p}$ and, for each $n, \Delta^{n+1} d_{p}=\Delta^{n} d_{p}-\Delta^{n} d_{p+1}$, then the Hausdorff matrix for the sequence $D$ is the infinite triangular matrix $[H(D)]$ such that $[H(D)]_{n p}=C_{n p} \Delta^{n-p} d_{p}, 0 \leqq p \leqq n$, and $[H(D)]_{n p}=0$ otherwise.

In considering the Riesz matrix for the sequence $A$ we may, without loss of generality, consider only sequences with $a_{0}=1$. It is well known that the Riesz matrix for the sequence $A$ is regular only in case the sequence of partial sums of $A$ diverges, and that the Hausdorff matrix for the sequence $D$ is regular only in case there exists a function, $\alpha$, of bounded variation on $[0,1]$, such that $\alpha(0+)=\alpha(0), \alpha(1)-\alpha(0)=1$ and for each nonnegative integer $p$, $d_{p}=\int_{0}^{1} t^{p} d \alpha(t)$.

It was noticed by Garabedian and Wall [1, pp. 198-199] that a certain class of hypergeometric summability matrices were also Riesz matrices. The following theorem establishes necessary and sufficient conditions that a Riesz matrix for the sequence $A$ also be a regular Hausdorff matrix.

THEOREM. If $A$ is a sequence such that $a_{0}=1$ and for each $p \geqq 1$, $a_{p} \geqq 0$ and $[A]$ is the Riesz matrix for $A$ then the following two statements are equivalent:

(1) There is a positive number $x$ such that $A=(1, x, x(x+1) / 2, \cdots$, $x(x+1) \cdots(x+p-1) / p !, \cdots)$.

(2) The matrix $[A]$ is a regular Hausdorff matrix.

Suppose (1) is true. The sequence of partial sums of $A$ is as follows; $S_{0}=1$ and if $p \geqq 1$ then $S_{p}=(1+x)(2+x) \cdots(p+x) / p$ !. A short computation will show that if each of $n$ and $p$ is a nonnegative integer, $p \leqq n$, then $[A]_{n p}=C_{n p}(x(n-p) ! /(x+p)(x+p+1) \cdots(x+n))$. In particular $[A]_{n n}=x /(x+n)$. An induction argument shows that if each of $n$ and $p$ is a nonnegative integer, $p \leqq n$, then $\Delta^{n}[A]_{p p}=(n !) x /(x+p)(x+p+1) \cdots(x+p+n)$. In particular

$$
C_{n p} \Delta^{n-p}[A]_{p p}=C_{n p}((n-p) ! x) /(x+p) \cdots(x+n)=[A]_{n p} .
$$

Received by the editors May 1, 1968. 
Therefore $[A]$ is a Hausdorff matrix. The function $\alpha(t)=t^{x}$ on $(0,1]$ with $\alpha(0)=0$ has the property that $\int_{0}^{1} t^{n} d \alpha(t)=x /(x+n)$, and hence $[A]$ is a regular matrix.

Suppose (2) is true. If $[A]$ is a Riesz matrix that is also a Hausdorff matrix then $[A]_{(n+1) n}=(n+1)\left([A]_{n n}-[A]_{(n+1)(n+1)}\right)$ for each $n$. Moreover $[A]_{(n+1) n}=a_{n} / S_{(n+1)}$ and the following recursion formula is obtained: $a_{n} / S_{n+1}=(n+1)\left(\left(a_{n} / S_{n}\right)-\left(a_{(n+1)} / S_{(n+1)}\right)\right)$. This may be solved for $a_{n+1}$ and we then obtain the formula $a_{n+1}=(n / n+1) a_{n}\left(S_{n} / S_{n-1}\right)$, which shows that the sequence $A$ is completely determined by the term $a_{1}$.

If $a_{1}=0$ then $A=(1,0,0,0, \cdots)$ and $[A]$ is not regular. It is, however, a semiregular Hausdorff matrix. Hence $a_{1}>0$. An induction argument shows that

$$
A=\frac{\left(1, a_{1}, a_{1}\left(1+a_{1}\right)\right.}{2}, \cdots, \frac{\left.a_{1}\left(1+a_{1}\right)\left(2+a_{1}\right) \cdots\left(n-1+a_{1}\right), \cdots\right)}{n !}
$$

This concludes the proof of the theorem.

Hausdorff has shown the summability method defined by a Riesz matrix of the type designated in the theorem is equivalent to $(C, 1)$ summability [1].

\section{REFERENCE}

1. H. L. Garabedian and H. S. Wall, Hausdorff methods of summation and continued fractions, Trans. Amer. Math. Soc. 48 (1940), 185-207.

Bowling Green State University 